\title{
Poster: Pallet Estimation for Food Bank Logistics Management
}

\author{
Alison Hau \\ Fei Fang \\ Zheyuan Ryan Shi \\ ahau@andrew.cmu.edu \\ feifang@cmu.edu \\ ryanshi@cmu.edu \\ Carnegie Mellon Univeresity \\ Pittsburgh, Pennsynvania, USA
}

\begin{abstract}
Food banks provide communities and organizations with food for those in need. One challenge they face is properly estimating the resources needed to fulfill orders. Estimating the number of shipping pallets needed for each order is an important step in allocating these resources, and coupled with limited data, provides a challenging mental task which the food bank staff grapple with on a daily basis. We provide an algorithm to estimate the number of pallets needed for an order based on the quantity of products, the known products-per-tier, and tiers-per-pallet values, as well as a scheme for testing this algorithm with limited data from the food bank. The algorithm aids in resource allocation by reducing uncertainty in the number of pallets needed.
\end{abstract}

\section{ACM Reference Format:}

Alison Hau, Fei Fang, and Zheyuan Ryan Shi. 2021. Poster: Pallet Estimation for Food Bank Logistics Management. In ACM SIGCAS Conference on Computing and Sustainable Societies (COMPASS) (COMPASS '21), June 28fuly 2, 2021, Virtual Event, Australia. ACM, New York, NY, USA, 4 pages. https://doi.org/10.1145/3460112.3471978

\section{INTRODUCTION}

In 2019, 10.5\% of American households faced food insecurity [3]. Community food banks, which stock food and offer it free of charge to individuals and charitable organizations, aim to assist food insecure households. Between March and June 2020, the beginning of the COVID-19 pandemic, food banks across the US distributed over 1.9 billion meals [11].

Food banks often distribute orders to partner organizations. When an order is placed, it is broken down into the requested products, which are collected from the warehouse and packaged onto pallets. Figure 1 illustrates how products are stacked on a pallet, before being packed onto trucks. The food bank then schedules delivery routes and assigns drivers. To do this, it is essential to know the number of pallets and trucks needed to deliver the day's orders so all deliveries are made on time. This is complicated by a number of uncertainties with the orders themselves.

Permission to make digital or hard copies of part or all of this work for personal or classroom use is granted without fee provided that copies are not made or distributed for profit or commercial advantage and that copies bear this notice and the full citation on the first page. Copyrights for third-party components of this work must be honored

For all other uses, contact the owner/author(s).

COMPASS '21, June 28-fuly 2, 2021, Virtual Event, Australia

(c) 2021 Copyright held by the owner/author(s).

ACM ISBN 978-1-4503-8453-7/21/06.

https://doi.org/10.1145/3460112.3471978

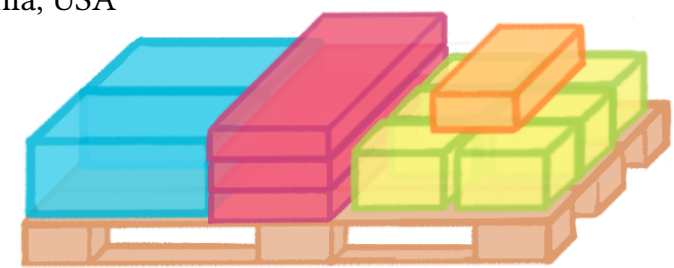

Figure 1: A visual of a pallet packed with items of different size.

The current process for estimating resources is to pack orders prior to delivery and record the number of pallets used. However, some pallets cannot be packed early, as food freshness and refrigeration capacity limit the space available for staging. Thus, it is necessary to estimate how many pallets will be needed. Food bank staff make estimates using heuristics based on product type, quantity, and packaging.

As demand at food banks increases, limitations in this process become more evident. One complication is uncertainty in the size of products. Items listed under one product number could have differing dimensions based on manufacturer and on supply date. The exact product dimensions are also unknown. This, plus the uncertainty of how human staff members will pack pallets, can lead to inaccurate estimation. Underestimating counts could lead to an order being sent under-filled or late. Overestimating could lead to wasted space on delivery trucks. Since it can be difficult to visualize combinations of products in 3D space to make these estimates, this is intuitively a problem better handled by an algorithmic approach. An automated process can estimate product dimensions from known product information more efficiently than a human and could lead to fewer under-filled orders and easier logistical planning.

We propose an algorithm to estimate how many pallets are needed for any given order to the food bank. First, we assume possible estimates of each product's dimensions based on the known quantity of product allowed per pallet tier and a pallet's dimensions. We pass all combinations of dimensions into a heuristic pallet-packing algorithm and use the results of the algorithm to provide the most likely number of pallets needed. To our knowledge, this is the first packing algorithm that does not rely on knowledge of exact product dimensions or on warehouse pallet-packers to exactly follow packing directions from the algorithm. 


\section{RELATED WORK}

Food banks have their presence in many parts of the world $[6,13]$. The logistics management of food bank supply chain and distribution is a popular topic of study. Martins et al. proposed a model to improve supply chain networking to collect and distribute donations [10]. Aleksandrov et al. investigated an online model to efficiently and fairly divide food bank resources amongst charitable organizations [2]. Other works address the packing of pallets prior to distribution. Ram summarizes a wide set of problems that fall under the umbrella of pallet-packing [12]. More recently, Al-Shayea developed a method to pack any number of mixed-size boxes onto 3D pallets to maximize pallet volume [1], and Gzara, et al. developed a method that further takes into consideration real-world limitations specific to pallet packing and moving [5]. However, these algorithms require that the bins or pallets are packed with rectangular items of known dimensions and do not account for the uncertainty of unknown product sizes. We expand on these previous works by including a product dimension estimation layer prior to pallet packing.

\section{ESTIMATING PRODUCT DIMENSIONS}

In this work, we focus on estimating the number of pallets needed for a given order. We first have to estimate possible dimensions of each product. The food bank does not keep data on the dimensions of each warehouse product. However, the food bank does log the weight; how many units can be packed on a single layer of a pallet, known as the TiValue; and how many layers of product are packed on one pallet, called the HiValue. The product audit log contains such information for 6942 distinct products. Occasionally, the audit contains more than one entry for a product because the product has multiple packagings over time. We keep all of them.

\subsection{Evaluating Possible Length, Width, Height Values}

We estimate the possible length, width, and height values for each product using all historical TiValue and HiValue entries from the audit logs. We collect and record the product identification, weight, TiValue, and HiValue of the entry.

We describe our procedure in Algorithm 1. We assume the TiValues from the audit logs, retrieved in line 2, are calculated from a uniform arrangement of products on a pallet tier, with the units arranged in a rectangle completely covering the pallet face. On line 3 we calculate all divisors of the TiValue. Each divisor corresponds to a possible configuration of products per our assumption. The divisor is the number of units arranged along the length of the pallet, and the TiValue divided by divisor is the number of units along the width, which we store in line 4 . On line 5, we divide the standard pallet length ( 40 inches) by the found products-perlength value and the standard pallet width (48 inches) by the found products-per-width values to find length and width for the product. On line 6, we then divide the maximum pallet height, 6 feet, by the HiValue to estimate the product height. We repeat for all log entries to get estimates for all known TiValue/HiValue pairs of a product. This workflow is illustrated in Fig. 2.

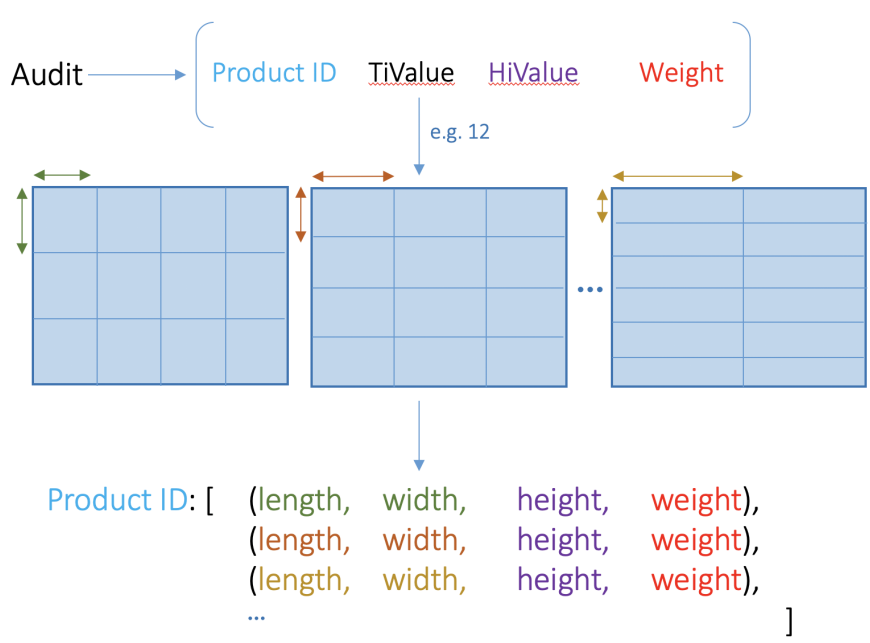

Figure 2: An example of dimension estimation workflow with TiValue of 12 .

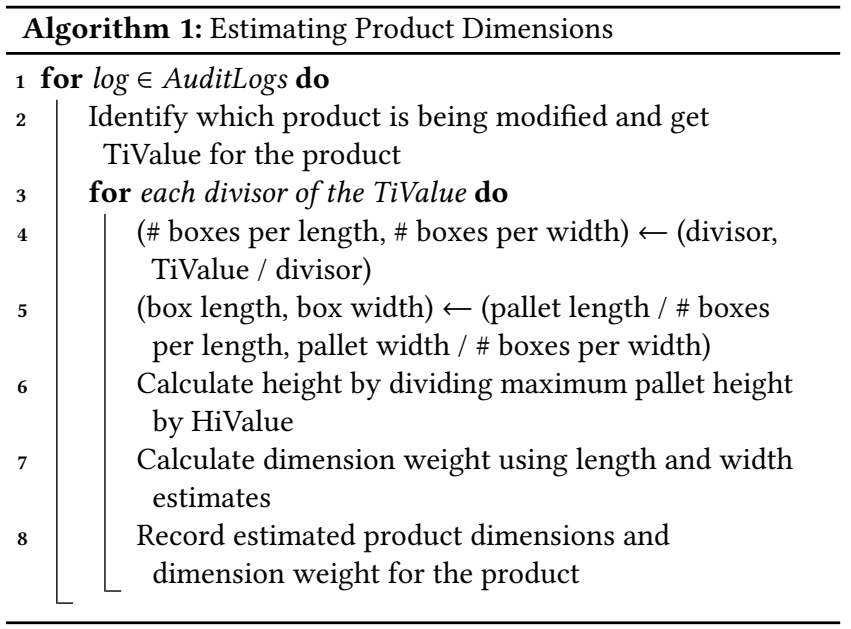

\subsection{Weighting Dimensions}

Not all divisors are equally likely to produce realistic product dimensions. Estimates of "long and skinny" dimensions are less likely to be evidenced in product packaging than estimates of similarlength sides. Thus, we assign a weight to each possible set of dimensions in line 8 of Algorithm 1. We calculate the estimate weight using three formulae. The Inverse Difference (ID) test group used weight $=\frac{1}{\mid \text { length-width } \mid}$, while the Inverse Difference Squared (IDS) group used weight $=\frac{1}{\mid \text { length-width| }\left.\right|^{2}}$, and the Min-Max $(\mathrm{MM})$ group used weight $=\frac{\min (\text { lengt } h, \text { width })}{\max (\text { length }, \text { width })}$.

We calculate the weights with the length and width estimations. This dimension weight, not to be confused with the product weight, is stored alongside the estimated dimensions.

\section{ESTIMATING PALLETS PER ORDER}

We describe our procedure in Algorithm 2. In the loop beginning on line 1 , we identify the products in the order, the quantities at which 


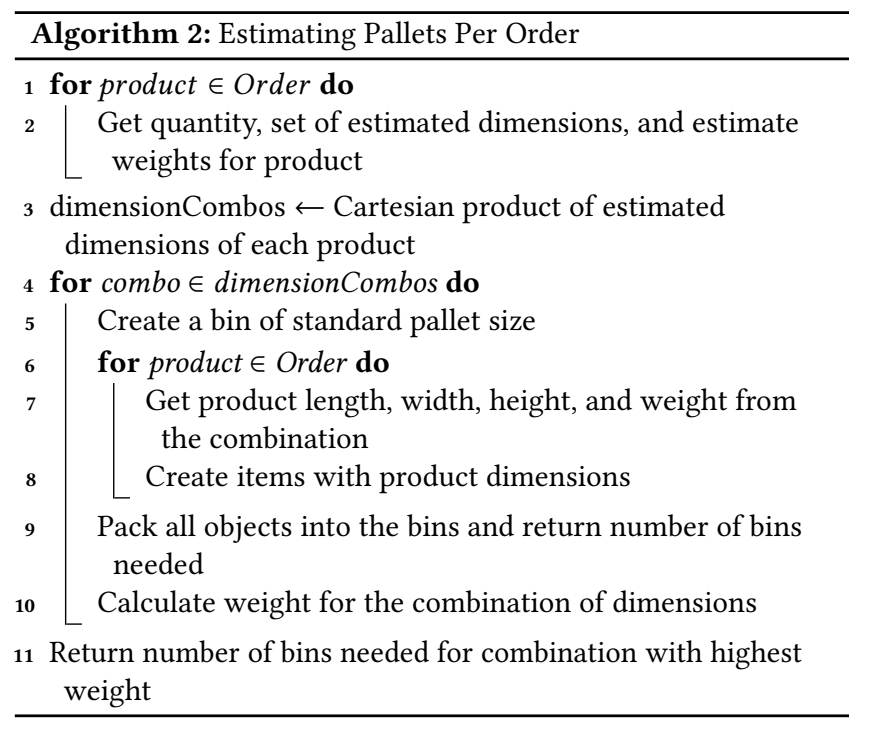

they were ordered, and the product's set of dimension estimates and estimate weights from the preprocessing step. In line 3, we take the Cartesian product of the sets to get all combinations of estimated dimensions. We then iterate through each combination of dimensions. We create a pallet-sized bin (40" x 48" by 72") in line 5 and an item for each product in the appropriate quantity, with the dimensions for specified in the combination in the loop starting on line 6. In line 9, these items are packed into the pallet using a modification of a pallet packing algorithm [8] based on the works of Li et al. [9] and Dube et al. [4]. The original algorithm maximizes the number of items packed into one bin by mathematically selecting locations to place each item and discarding items deemed unable to fit within the bin. We modify this to allow multiple bins, first trying to place an item into existing bins before creating a new bin if the existing bins cannot fit the item. This allows us to use the heuristic bin packing algorithm without limiting the simulation to one pallet. We return the number of pallets needed to pack all items. In line 10 , we calculate the estimate-weight of the combination, which reflects the likelihood of the combination of product dimensions, by taking the mean of all products' estimate-weights. In line 11, we report the estimate with the highest weight.

\section{EXPERIMENTATION}

In our experiments, we wanted to evaluate our pallet estimation algorithm. Our dataset contained orders from 2 May 2018 to 24 April 2020, and we generated a pallet estimation for each order. However, the food bank did not log the actual number of pallets used by each order, so we needed to generate the ground truth data against which we would compare our algorithm.

\subsection{Synthetic Testing Dataset}

For each product, we passed the name of the product into an Amazon web scraper [7], which searched the product on Amazon and retrieved product dimension information of the most popular search result. We performed a manual check that each scraped dimension was reasonable to the Ti- and Hi- Values of the product in the food bank data. Values that did not produce similar Ti- and Hi- Values or did not return results from the scrape were disregarded. These products were typically either fresh produce/meat or food bank-specific items, which tend to lack standardized packing dimension.

These results were then associated with the product and stored for reference. We treated this dimension information as the actual dimension. Then, for each order we ran the pallet packing algorithm to get the number of pallets needed. This became our ground truth results. The testing dataset contained 1272 products, including the top 800 most frequently ordered items at the food bank.

\subsection{Experimentation Groups and Metrics}

Experiments were conducted on five algorithms to compare performance. Three versions of the estimation algorithm with different dimension weight formulas were compared to two benchmarks. The Upper Bound (UB) benchmark group made estimates by multiplying TiValue and HiValue to get a number of products allowed on a pallet, then dividing the product quantity by the number allowed per pallet and rounding to the next integer. This provides an upper bound on number of pallets by assuming that one pallet can only contain one product type. The Naive benchmark group estimated by dividing the product quantity by the number of product allowed per pallet to get a fractional number of pallets needed for the product, then adding all fractions of pallets needed in the order. This naively assumes we can combine any fractions of pallets arbitrarily.

The metrics used for assessing these algorithms were percent accuracy and root mean squared error (RMSE), calculated using the following formulas:

$$
\begin{gathered}
\% \text { Accuracy }=\frac{\# \text { correct }}{\# \text { test cases }} \\
\text { RMSE }=\frac{1}{\# \text { test cases }} \sqrt{\sum_{\text {test cases }}(\text { estimated }- \text { actual })^{2}}
\end{gathered}
$$

The percent-accuracy metric measures percentage of orders estimated accurately, which tells us how reliable the food bank should expect the algorithm to be. RMSE tells us how many pallets we over- or under-estimated by on average, which is useful to evaluate mis-allocated resources per order using the algorithm.

\subsection{Results}

We tested on orders that only contained products in our synthetic testing dataset. This totalled to 1298 orders, which we analyzed as two groups: 1203 small orders, consisting of 1 pallet, and 95 large orders, consisting of $2+$ pallets.

As shown in Figures 3 and 4, the Inverse Difference group had $97.12 \%$ accuracy and 0.4259 root mean squared error for small orders and $85.46 \%$ accuracy and 1.32 RMSe for large orders. The Inverse Difference Squared group also had $97.12 \%$ accuracy and 0.4259 RMSE for small orders and $85.46 \%$ accuracy and 1.32 RMSE for large orders, while the Min-Max group had slightly lower accuracy for both types of orders, with $96.76 \%$ and RMSE of 0.5026 pallets for small orders and $83.98 \%$ accuracy and 1.11 RMSE for large orders. This compares to the two benchmark groups - the Naive Estimate group had $58.08 \%$ accuracy and RMSE of 5.00 for small orders and $3.97 \%$ accuracy and 1.42 RMSE for large orders; the Upper Bound group had $36.72 \%$ accuracy and 7.58 RMSE for small orders and $37.54 \%$ accuracy 1.69 root mean squared error for large orders. 


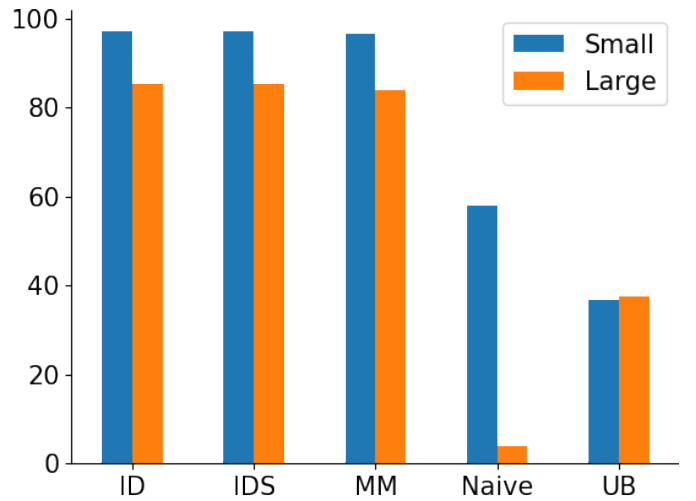

Figure 3: Percent Accuracy for each testing group

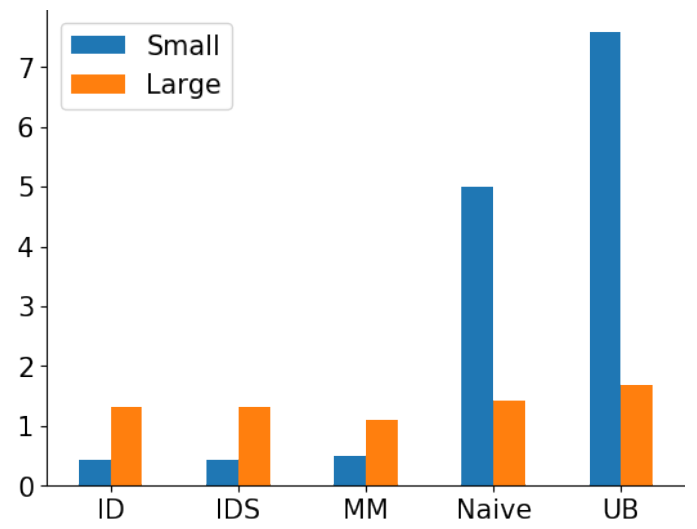

Figure 4: RMSE for each testing group

\section{CONCLUSION AND DISCUSSION}

We designed an algorithm to improve food bank logistics management by estimating the number of pallets required to fulfil a given order. The algorithm correctly estimates for $97.12 \%$ of small historical orders and $85.46 \%$ of large historical orders. Using this tool, a pallet-packing warehouse with items of unknown size could improve logistical planning and avoid mis-allocating resources.

Further evaluation of this algorithm would require other sources for ground truth data. Other food banks or similar organizations may keep data useful for comparing ground truth number of pallets needed per order to the estimation from the algorithm. This sort of data would include each order's products, each pallet stacking info (HiValue, TiValue, and weight), and the actual number of pallets the warehouse staff needed to fulfil the order.

Throughout this work, we intend for the algorithm to assist any human labor at the food bank rather than replace. Although we use a bin-packing algorithm, we do not manage how the workers pack the pallets on the ground. As a preliminary study, the presented work is limited due to the lack of ground truth data. We are working with our partner to gather such data in the near future.

\section{ACKNOWLEDGMENTS}

This work was supported by NSF grant IIS-1850477 and IIS-2046640 (CAREER).

\section{REFERENCES}

[1] Adel Mohammed Al-Shayea. 2011. Solving the Three-Dimensional Palet-Paking Problem Using Mixed 0 - 1 Model. Journal of Service Science and Management (11 2011).

[2] Martin Aleksandrov, Haris Aziz, Serge Gaspers, and Toby Walsh. 2015. Online Fair Division: analysing a Food Bank problem. arXiv:1502.07571 [cs.GT]

[3] Alisha Coleman-Jensen, Matthew P. Rabbitt, Christian A. Gregory, and Anita Singh. 2020. Household Food Security in the United States in 2019. Technical Report ERR-275. U.S. Department of Agriculture.

[4] Erick Dube, Leon Kanavathy, Leon K@i, and Owave Za. 2006. Optimizing ThreeDimensional Bin Packing Through Simulation. (01 2006).

[5] Fatma Gzara, Samir Elhedhli, and Burak C. Yildiz. 2020. The Pallet Loading Problem: Three-dimensional bin packing with practical constraints. European Journal of Operational Research 287, 3 (2020), 1062-1074. https://doi.org/10.1016/ j.ejor.2020.04.053

[6] Becky Handforth, Monique Hennink, and Marlene B. Schwartz. 2013. A Qualitative Study of Nutrition-Based Initiatives at Selected Food Banks in the Feeding America Network. Fournal of the Academy of Nutrition and Dietetics 113, 3 (2013), 411-415. https://doi.org/10.1016/j.jand.2012.11.001

[7] Scrape Hero. 2020. Amazon Scraper using Selectorlib. https:/github.com/ scrapehero-code/amazon-scraper.

[8] Janet-19. 2020. 3D-Bin-Packing-Problem. https://github.com/Janet-19/3d-binpacking-problem.

[9] Xueping Li, Zhaoxia Zhao, and Kaike Zhang. 2014. A genetic algorithm for the three-dimensional bin packing problem with heterogeneous bins. IIE Annual Conference and Expo 2014.

[10] C.L. Martins, M.T. Melo, and M.V. Pato. 2019. Redesigning a food bank supply chain network in a triple bottom line context. International fournal of Production Economics 214 (2019), 234-247. https://doi.org/10.1016/j.ijpe.2018.11.011

[11] Paul Morello. 2020. The first months of the food bank response to COVID, by the numbers. https://www.feedingamerica.org/hunger-blog/first-months-foodbank-response-covid-numbers.

[12] Balasubramanian Ram. 1992. The pallet loading problem: A survey. International Journal of Production Economics 28, 2 (1992), 217-225. https://doi.org/10.1016/ 0925-5273(92)90034-5

[13] Valerie Tarasuk, Andrée-Anne Fafard St-Germain, and Rachel Loopstra. 2020. The Relationship Between Food Banks and Food Insecurity: Insights from Canada. VOLUNTAS: International fournal of Voluntary and Nonprofit Organizations 31, 5 (2020), 841-852. https://doi.org/10.1007/s11266-019-00092-w 